\title{
Chest Pain Due to Pericardial Effusion as Initial Presenting Feature of Rheumatoid Arthritis: Case Report and Review of the Literature
}

\author{
Malahat Movahedian ${ }^{\mathrm{a}, \mathrm{c}}$, Wais Afzal ${ }^{\mathrm{a}}$, Tannaz Shoja ${ }^{\mathrm{a}}$, Kelly Cervellione ${ }^{\mathrm{b}}$, \\ Jebun Nahar ${ }^{\mathrm{a}}$, Katerina Teller ${ }^{\mathrm{a}}$
}

\begin{abstract}
Pericardial effusions are not uncommon in rheumatoid arthritis (RA); however, they are rarely the presenting symptom of the disease. We describe a 55-year-old female who presented to the emergency department with complaints of chest pain and dyspnea on exertion. Initial workup revealed a medium-sized pericardial effusion. The wide spectrum of etiologies, including infectious and non-infectious disease, was explored. Eventually, after ruling out an array of disease states, rheumatologic workup was positive for RA. The initial presentation in our case was atypical due to absence of small joint polyarthritis and other common symptoms of RA. In difficult cases, extensive workup including laboratory tests, electrocardiography, echocardiography and imaging studies can aid in narrowing the causes of pericardial effusion. This case demonstrates that pericardial effusion could be an early presenting feature of RA, even in the absence of more common symptoms, and should be considered in differential diagnosis.
\end{abstract}

Keywords: Rheumatoid arthritis; Pericardial effusion; Pericarditis; Rheumatoid factor; Anti-CCP antibody

\section{Introduction}

Rheumatoid arthritis (RA) is a systemic autoimmune disorder of unknown cause that typically presents as a systemic inflammatory polyarthritis, but can also have accompanying fever, weight loss, and fatigue. Nearly half of patients with RA have additional non-joint involvement, including skin, eyes, lungs, and heart. Cardiac manifestations are rarely the presenting symptom of RA. Pericarditis is the most frequent cardiac

Manuscript submitted May 27, 2017, accepted June 16, 2017

${ }^{a}$ Department of Internal Medicine, Jamaica Hospital Medical Center, 8900 Van-Wyck Expressway, Jamaica, NY 11418, USA

bDepartment of Clinical Research, Jamaica Hospital Medical Center, 8900 Van-Wyck Expressway, Jamaica, NY 11418, USA

${ }^{\mathrm{c} C o r r e s p o n d i n g ~ A u t h o r: ~ M a l a h a t ~ M o v a h e d i a n, ~ D e p a r t m e n t ~ o f ~ I n t e r n a l ~ M e d i-~}$ cine, Jamaica Hospital Medical Center, 8900 Van Wyck Expressway, Jamaica, NY 11418, USA. Email: malahat.movahedian@gmail.com

doi: https://doi.org/10.14740/cr570e manifestation, typically developing with progression of the disease, and effecting approximately $40 \%$ of patients [1-3]. Here we describe a patient who presented initially with chest pain due to pericardial effusion. After extensive workup, she was found to have RA without any other potential causes of pericardial effusion.

\section{Case Report}

A 55-year-old African-American female presented with constant, non-radiating, left-sided chest pain of moderate intensity for 3 days. There was no associated nausea, vomiting, palpitations and diaphoresis; however, she mentioned that it worsened with deep breathing, coughing and lying on her sides. She also reported shortness of breath on exertion. She denied fever, chills, upper respiratory infection, shortness of breath at rest and skin rash. She denied joint pain except for chronic knee pain due to osteoarthritis. She reported no sick contacts or recent travel out of the country. She had a similar episode of chest pain 5 months prior, for which she was hospitalized, but initial cardiac workup at that time were non-diagnostic and pain subsided with pain medications. Our patient reported a history of hypertension, pre-diabetes, anemia, obesity, osteoarthritis, and hypothyroidism. She did not use tobacco or alcohol. Medications included lisinopril, amlodipine, ferrous sulfate and levothyroxine. Her family history was positive for myocardial infarction and RA in her mother and hypertension in her father and mother.

Physical examination was significant for decreased breath sounds and cardiac friction rub. There was no reproducible chest pain. Routine labs were normal. Electrocardiography (ECG) and cardiac markers were normal. Endocrine labs were unrevealing. Chest X-ray (CXR) demonstrated cardiomegaly (Fig. 1a), which was not present on X-ray 5 months prior (Fig. 1b).

Further evaluation by CT pulmonary angiography (CTPA) revealed a large-sized pericardial effusion with mild left-sided pleural effusion (Fig. 2).

Echocardiography revealed ejection fraction (EF) of $66 \%$ and a moderate-sized pericardial effusion was identified circumferential to the heart (Fig. 3). There was no significant chamber collapse. Features were not consistent with cardiac tamponade. A left pleural effusion also was detected. 

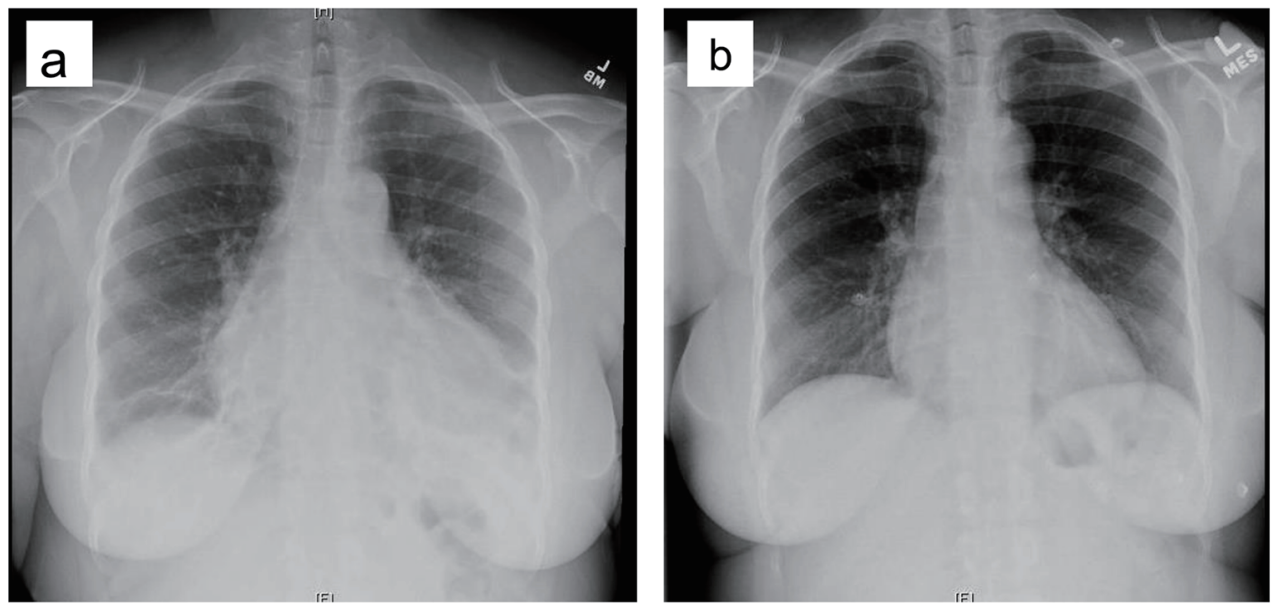

Figure 1. (a) CXR of the patient in current admission.

(b) CXR of the patient 5 months prior to this admission.

The patient was started on ibuprofen $600 \mathrm{mg}$, three times a day, colchicine $0.6 \mathrm{mg}$ daily and low-dose furosemide. Based on history and unrevealing tests, autoimmune and connective tissue disease panel were collected (Table 1). Results included negative rheumatoid factor (RF), elevated erythrocyte sedimentation rate (ESR), elevated C-reactive protein (CRP) and strongly positive anti-cyclic citrullinated peptide (CCP) (laboratory reference value $>59$, strong positive).

The echocardiography repeated 1 month later after treatment (Fig. 4) revealed EF of 65\% and minimal pericardial effusion. Patient's symptoms in terms of chest pain and shortness of breath improved significantly.

Post-discharge follow-up 3 months later revealed new onset bilateral wrist pain and morning stiffness of unspecified duration. Pain was partially responsive to NSAIDs. The X-ray of the wrist revealed joint spaces were maintained. No erosive

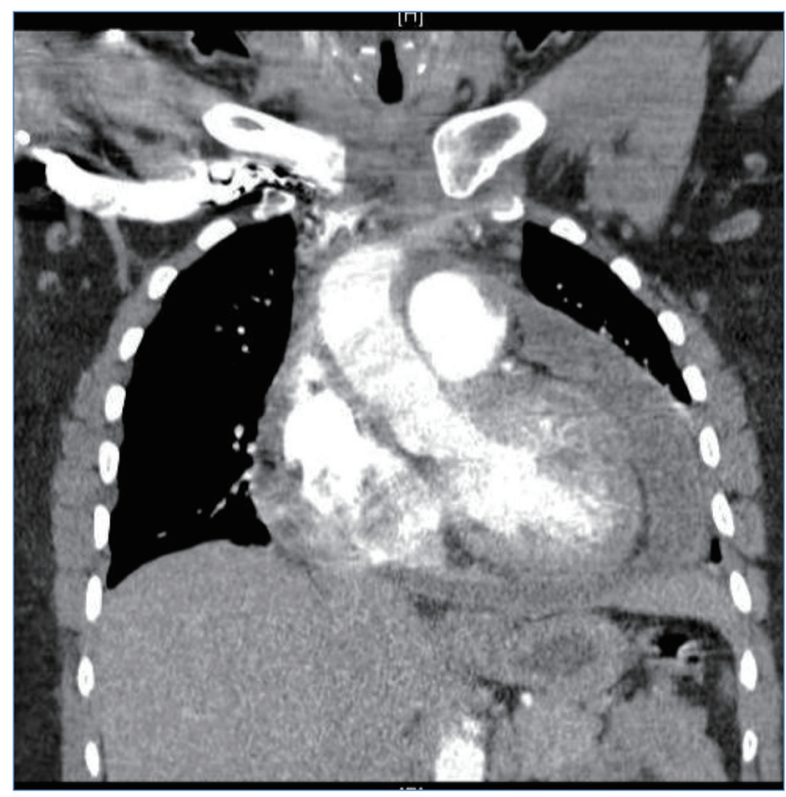

Figure 2. CT pulmonary angiography (CTPA). change was identified. Patient was started on prednisone 10 $\mathrm{mg}$ daily. She was advised to do follow-up in rheumatology clinic for early detection of erosive changes.

\section{Discussion}

Pericarditis is a well-described entity in patients with RA, with reports dating back nearly 120 years (Bannatyne 1898). However, extra-articular manifestations of the disease, including pericarditis, are rarely the presenting signs of undiagnosed RA. The review of literature shows few cases of RA with similar presentation of our case. Pericarditis is the most frequent cardiac manifestation of RA, affecting up to half of patients [1-3]. Most cases of pericarditis are asymptomatic and are revealed

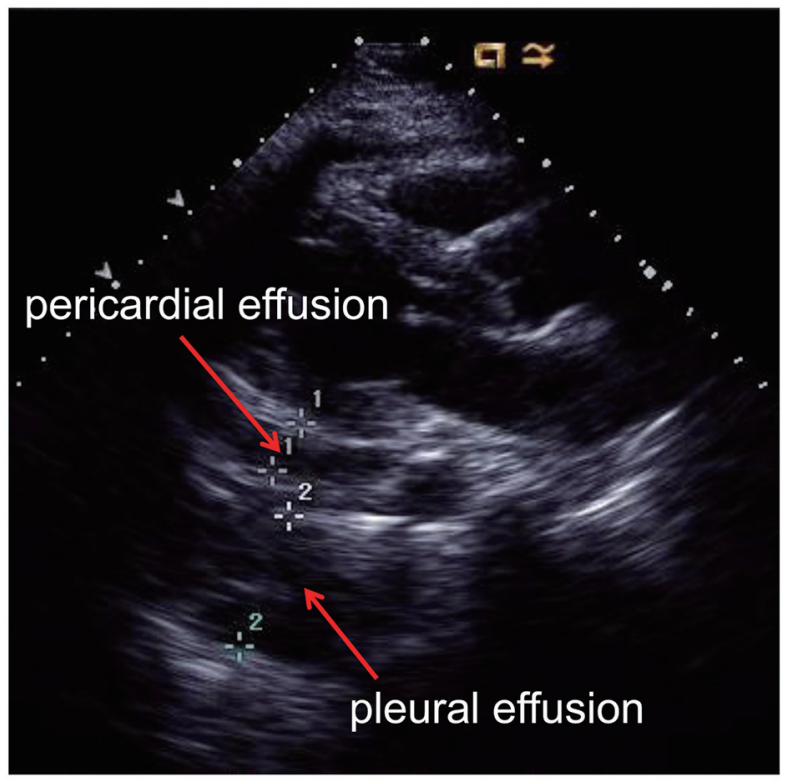

Figure 3. 2D echocardiogram revealing a medium-sized pericardial effusion and a small-sized pleural effusion. 
Table 1. Autoimmune and Connective Tissue Disease Panel

\begin{tabular}{lllllll} 
Test & RF & Anti-CCP & ANA & Anti-ds-DNA & ESR & CRP \\
\hline Reference range & Negative & $<20 \mathrm{U}$ & Negative & $<4 \mathrm{IU} / \mathrm{mL}$ & $0-30$ & $0.70-1.00$ \\
Result & Negative & $>250 \mathrm{U}$ & Negative & $<4 \mathrm{IU} / \mathrm{mL}$ & 93 & 8.90 \\
\hline
\end{tabular}

incidentally or on autopsy. Less than $10 \%$ of cases are symptomatic, usually occurring in patients with nodular disease or in patients who are highly RF positive [4]. Symptomatic pericarditis is associated with higher mortality in patients with RA [3, 5]. Additional pericardial manifestations of RA include cholesterol pericarditis $[6,7]$, effusive-constrictive pericarditis $[8,9]$, and cardiac tamponade $[10,11]$. These have been described in newly diagnosed cases as well as long-standing RA.

In patients who present with pericardial effusion where there is no known cause based on history and no obvious infectious etiology, RA should be ruled out. For serologic diagnosis, presence of autoantibodies, including RF and anti-CCP antibodies, should be explored. Presence of autoantibodies can precede clinical disease by years. Early diagnosis and treatment may prevent joint destruction and suppress disease progression. Anti-CCP antibodies occur less frequently than RF, but have a higher diagnostic specificity. Anti-CCP antibodies are directed against citrullinated proteins, including proteins present in inflamed joint tissues. Clinically, the presence of anti-CCP antibodies predicts a greater risk of erosive disease [12].

Aspirin or NSAIDs remain the mainstay of treatment for pericarditis. NSAIDs should be administered at appropriate anti-inflammatory dosages (e.g. aspirin at 2 - 4 g daily, indomethacin at $75-150 \mathrm{mg}$ daily, and ibuprofen at 1,600-3,200 mg daily). These treatments should be continued until complete normalization of CRP. Corticosteroids can be used in recurrent pericarditis but evidence is weak in supporting this treatment $[2,7,13]$. Colchicine $2 \mathrm{mg} /$ day for 1 - 2 days, followed by a maintenance dose of $1 \mathrm{mg} /$ day, has been recommended in some studies to treat recurrent pericarditis and is

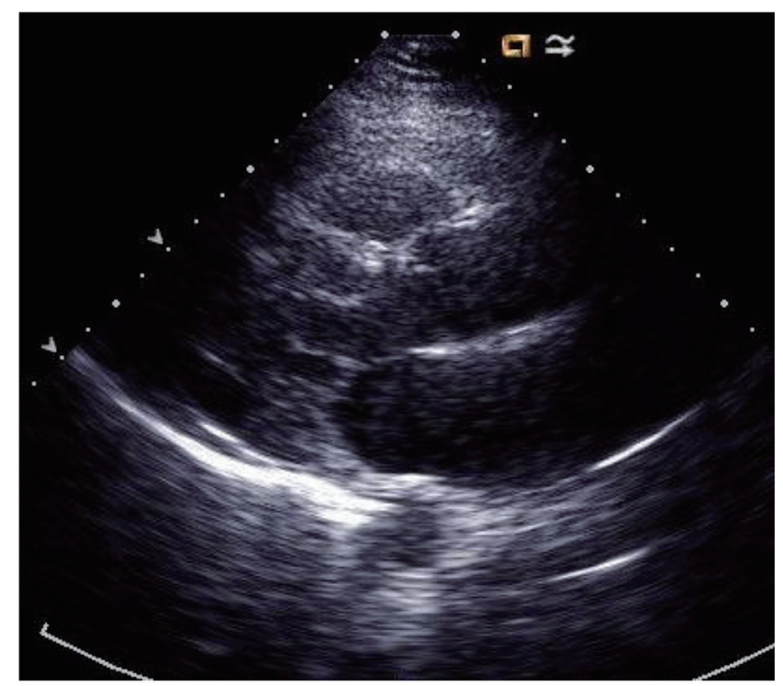

Figure 4. 2D echocardiogram repeated 1 month after treatment demonstrates resolving pericardial effusion. considered useful in acute pericarditis [13].

Severe cases of RA-related pericarditis can be associated with cardiac tamponade, hemodynamically significant pericardial effusion, and constrictive or effusive-constrictive physiology. RA-related pericarditis can also be treatment refractory $[1,14,15]$. When a significant pericardial effusion is present, diagnostic pericardiocentesis is recommended. Pericardiocentesis may also be considered for large or symptomatic effusions refractory to medical treatment [13]. Other invasive treatments include pericardiectomy and pericardiotomy [13].

\section{Disclosure}

The manuscript, patient data, and imaging are reproduced in accordance with Jamaica Hospital Medical Center policies approved by internal IRB.

\section{Conflicts of Interest}

The authors declare that there are no conflicts of interest.

\section{References}

1. Mankad R, Ball CA, Myasoedova E, Matteson EL. Nonatherosclerotic cardiac manifestations of rheumatoid arthritis. In: Semb AG (ed.) Handbook of cardiovascular disease management in rheumatoid arthritis. Springer International Publishing; 2017.

2. Corrao S, Messina S, Pistone G, Calvo L, Scaglione $\mathrm{R}$, Licata $\mathrm{G}$. Heart involvement in rheumatoid arthritis: systematic review and meta-analysis. Int J Cardiol. 2013;167(5):2031-2038.

3. Hara KS, Ballard DJ, Ilstrup DM, Connolly DC, Vollertsen RS. Rheumatoid pericarditis: clinical features and survival. Medicine (Baltimore). 1990;69(2):81-91.

4. Jurik AG, Graudal H. Pericarditis in rheumatoid arthritis. A clinical and radiological study. Rheumatol Int. 1986;6(1):37-42.

5. Mitchell DM, Spitz PW, Young DY, Bloch DA, McShane DJ, Fries JF. Survival, prognosis, and causes of death in rheumatoid arthritis. Arthritis Rheum. 1986;29(6):706714.

6. Qian LY, Pang J, Che XD, Ding YH, Zou H, Zhou B. Cholesterol pericarditis associated with rheumatoid arthritis: A rare case report. Medicine (Baltimore). 2016;95(44):e5305.

7. Gecmen C, Gecmen GG, Kahyaoglu M, Ece D, Ozkan B, Karaman O, Naser A, et al. Pericardial effusion due 
to cholesterol pericarditis in case of rheumatoid arthritis. Echocardiography. 2016;33(10):1614-1616.

8. Schatz A, Trankle C, Yassen A, Chipko C, Rajab M, Abouzaki N, Abbate A. Resolution of pericardial constriction with Anakinra in a patient with effusive-constrictive pericarditis secondary to rheumatoid arthritis. Int J Cardiol. 2016;223:215-216.

9. Adamson VW, Slim JN, Leclerc KM, Slim AM. A rare case of effusive constrictive cholesterol pericarditis: a case report and review. Case Rep Med. 2013;2013:439505.

10. Al-Ani M, Weber M, Winchester D, Kosboth M. Pericardial mass in a patient with rheumatoid arthritis. BMJ Case Rep. 2015;2015.

11. Soh MC, Hart HH, Corkill M. Pericardial effusions with tamponade and visceral constriction in patients with rheu- matoid arthritis on tumour necrosis factor (TNF)-inhibitor therapy. Int J Rheum Dis. 2009;12(1):74-77.

12. Niewold TB, Harrison MJ, Paget SA. Anti-CCP antibody testing as a diagnostic and prognostic tool in rheumatoid arthritis. QJM. 2007;100(4):193-201.

13. Imazio M, Spodick DH, Brucato A, Trinchero R, Adler Y. Controversial issues in the management of pericardial diseases. Circulation. 2010;121(7):916-928.

14. Langley RL, Treadwell EL. Cardiac tamponade and pericardial disorders in connective tissue diseases: case report and literature review. J Natl Med Assoc. 1994;86(2):149153.

15. Yousuf T, Kramer J, Kopiec A, Bulwa Z, Sanyal S, Ziffra J. A rare case of cardiac tamponade induced by chronic rheumatoid arthritis. J Clin Med Res. 2015;7(9):720-723. 